Supporting Information For

\title{
Structural Diversity and Incompatibility Induced Complex Phase Formation Behavior in the Stuffed Tridymites $\mathrm{Ca}_{1-x} \mathrm{Sr}_{x} \mathrm{Ga}_{2} \mathrm{O}_{4}$
}

Yuhang Chen, ${ }^{\dagger}$ Jia Yang,, ${ }^{\#}$ Pengfei Jiang,,${ }^{*} \dagger$ Fuwei Jiang, ${ }^{\dagger}$ Hengwei Wei,,${ }^{\ddagger}$ Wenliang Gao, ${ }^{\dagger}$ Rihong Cong, ${ }^{\dagger}$ Tao Yang ${ }^{* `} \uparrow$

$\dagger$ College of Chemistry and Chemical Engineering, Chongqing University, Chongqing, 401331, P. R.

China

\# School of Chemistry and Chemical Engineering, Yangtze Normal University, Chongqing, 408100, P. R. China

$¥$ Basic Experimental Teaching Centre, Shaanxi Normal University, Xi’an, 710062, Shaanxi Province, P. R. China

*Corresponding authors: pengfeijiang@cqu.edu.cn; taoyang@cqu.edu.cn. 

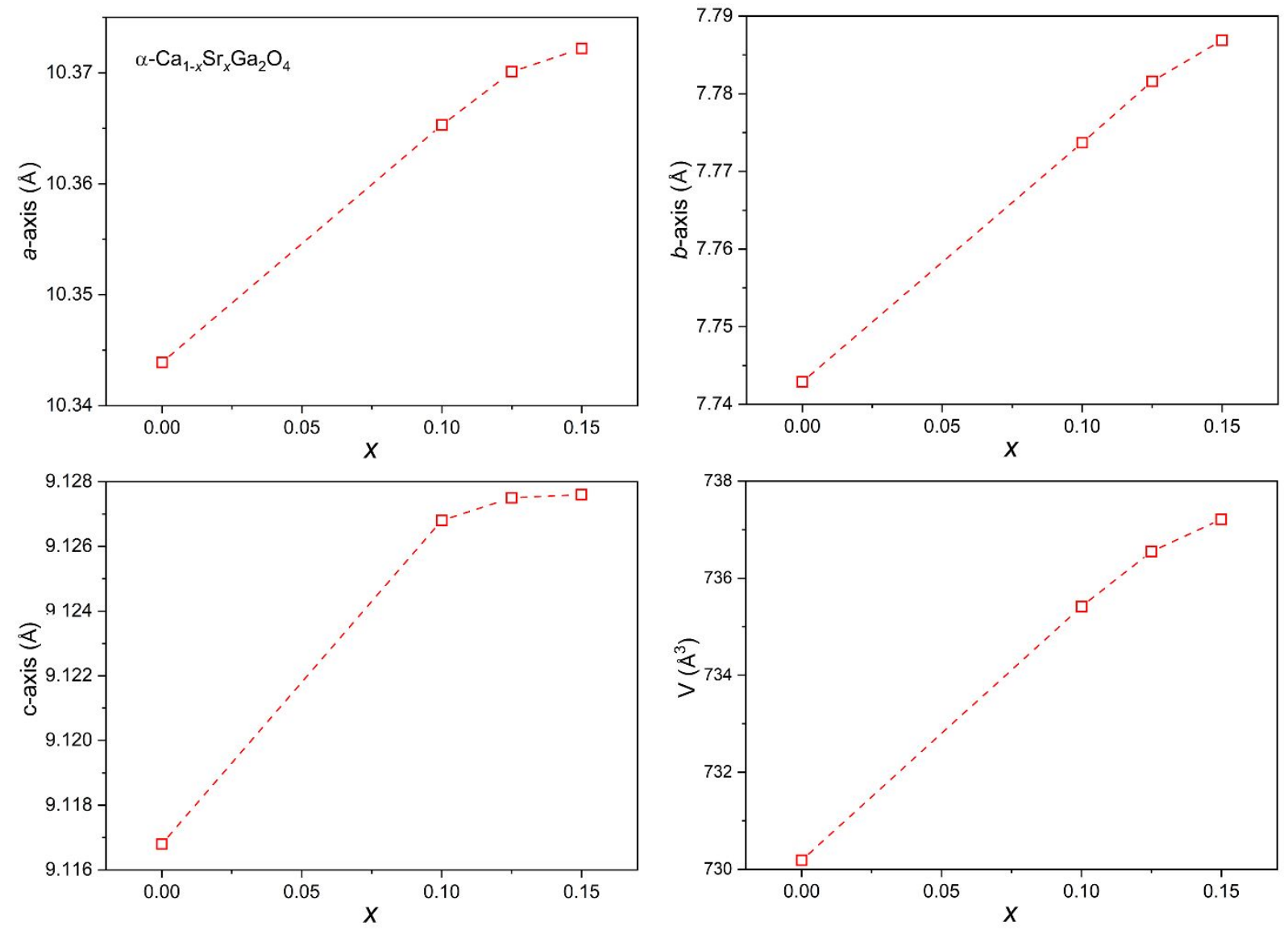

Figure S1. Plots of lattice parameters for $\alpha$ - $\mathrm{CaGa}_{2} \mathrm{O}_{4}$-type phases against $\mathrm{Sr}^{2+}$-content in $\mathrm{Ca}_{1-}$ ${ }_{x} \mathrm{Sr}_{x} \mathrm{Ga}_{2} \mathrm{O}_{4}(0 \leq x \leq 0.15)$.

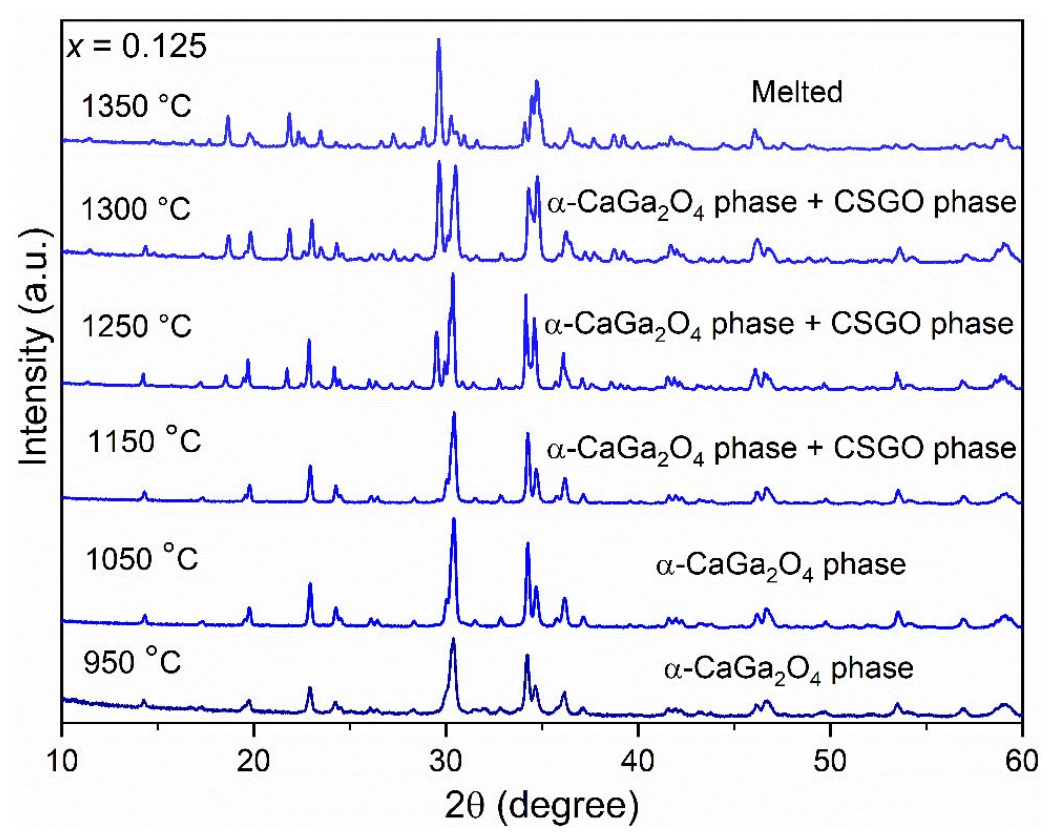


Figure S2. XRD patterns for $\mathrm{Ca}_{1-x} \mathrm{Sr}_{x} \mathrm{Ga}_{2} \mathrm{O}_{4}$ with $x=0.125$ after being calcinated at different temperatures.
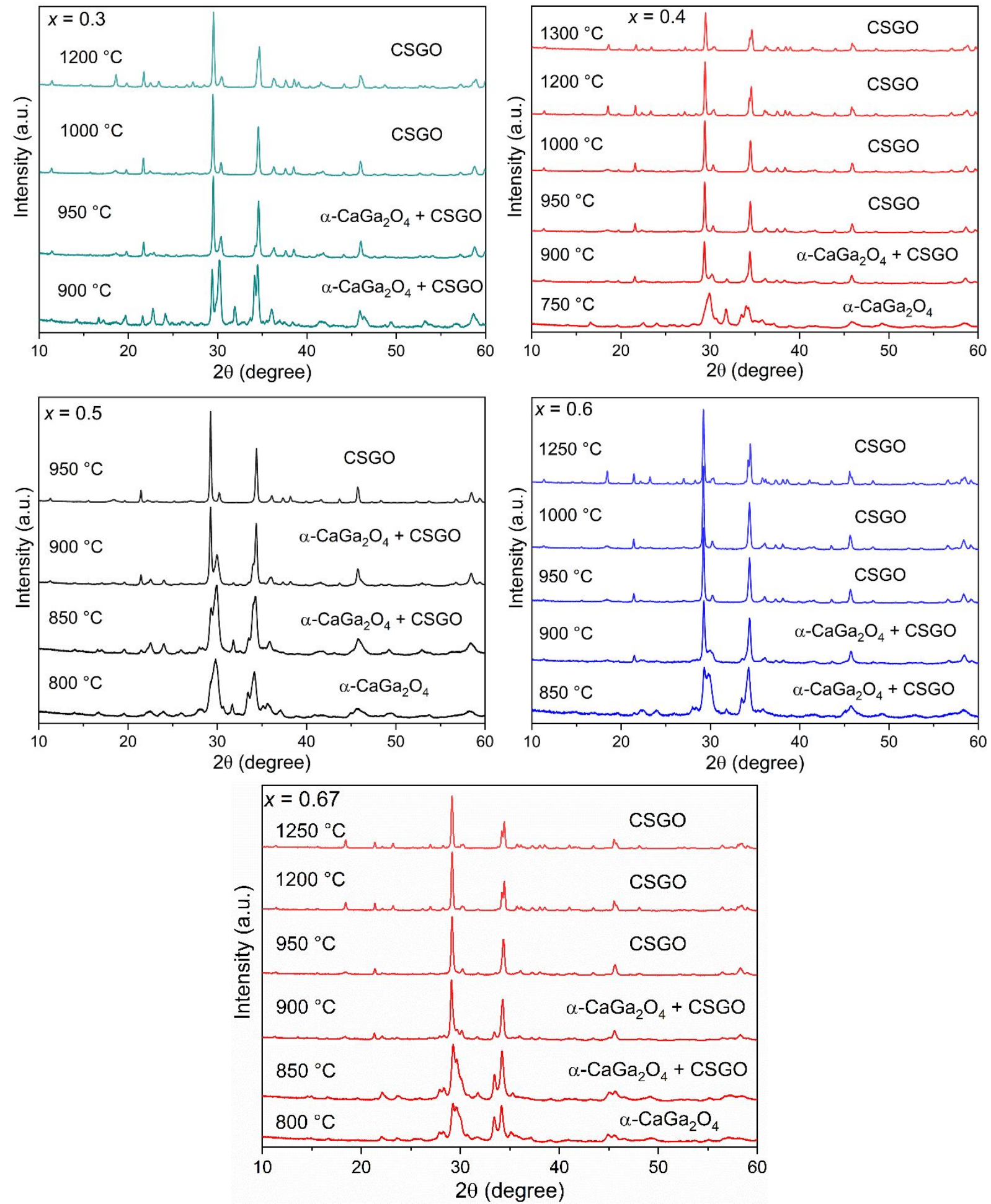

Figure S3. XRD patterns for $\mathrm{Ca}_{1-x} \mathrm{Sr}_{x} \mathrm{Ga}_{2} \mathrm{O}_{4}(x=0.3,0.4,0.5,0.6$, and 0.67) after being heated at different temperatures. 

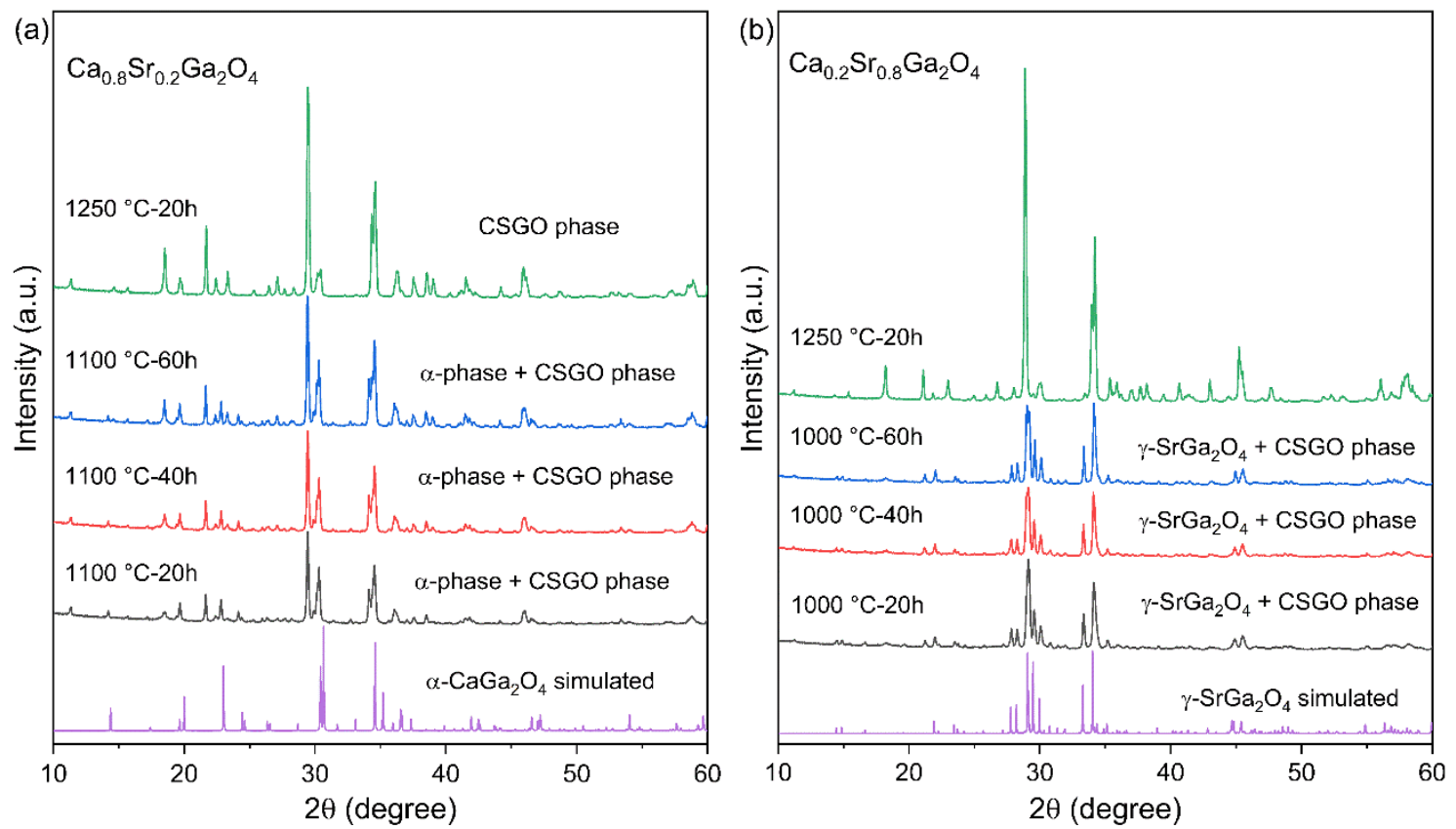

Figure S4. PXRD patterns for $\mathrm{Ca}_{1-x} \mathrm{Sr}_{x} \mathrm{Ga}_{2} \mathrm{O}_{4}(x=0.2$ and 0.8$)$ after being heated at the temperature within the biphasic region for different dwell times. 

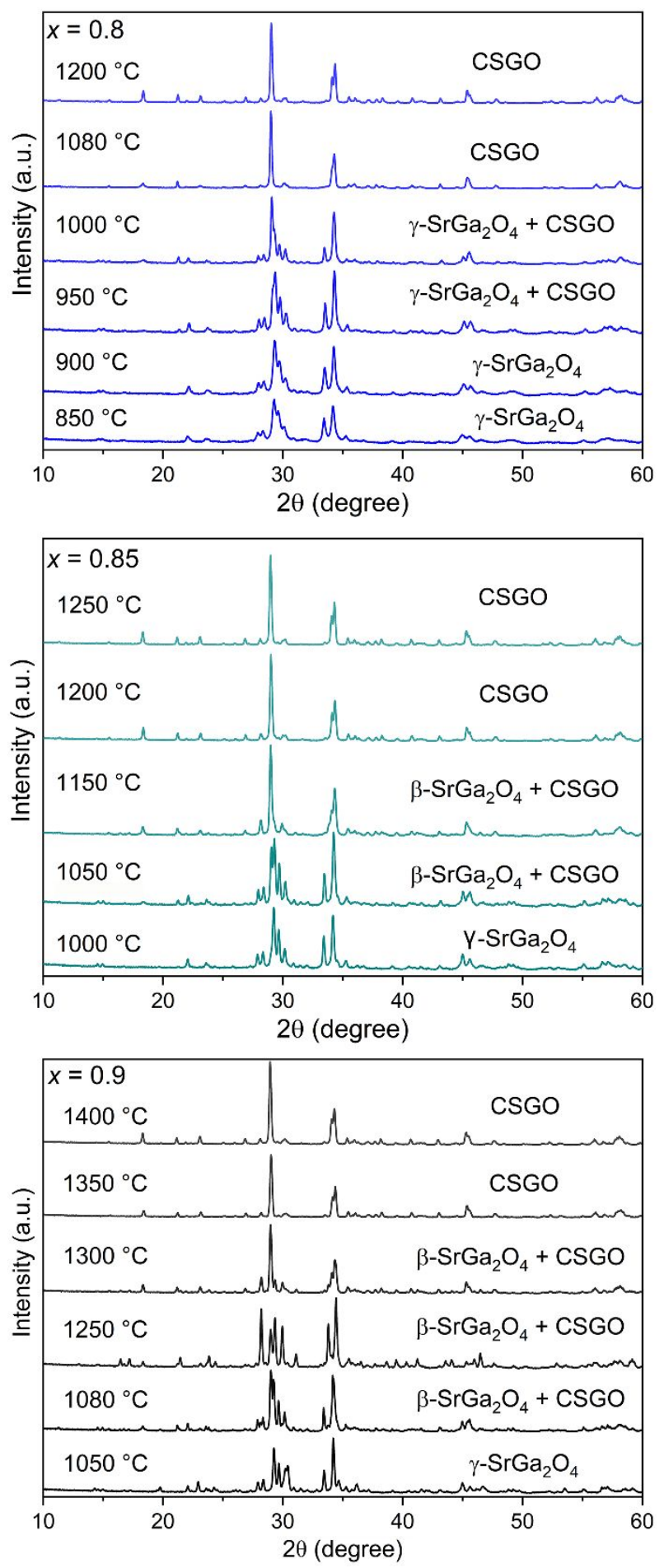

Figure S5. XRD patterns for $\mathrm{Ca}_{1-x} \mathrm{Sr}_{x} \mathrm{Ga}_{2} \mathrm{O}_{4}(x=0.8,0.85$, and 0.9) after being heated at different temperatures. 

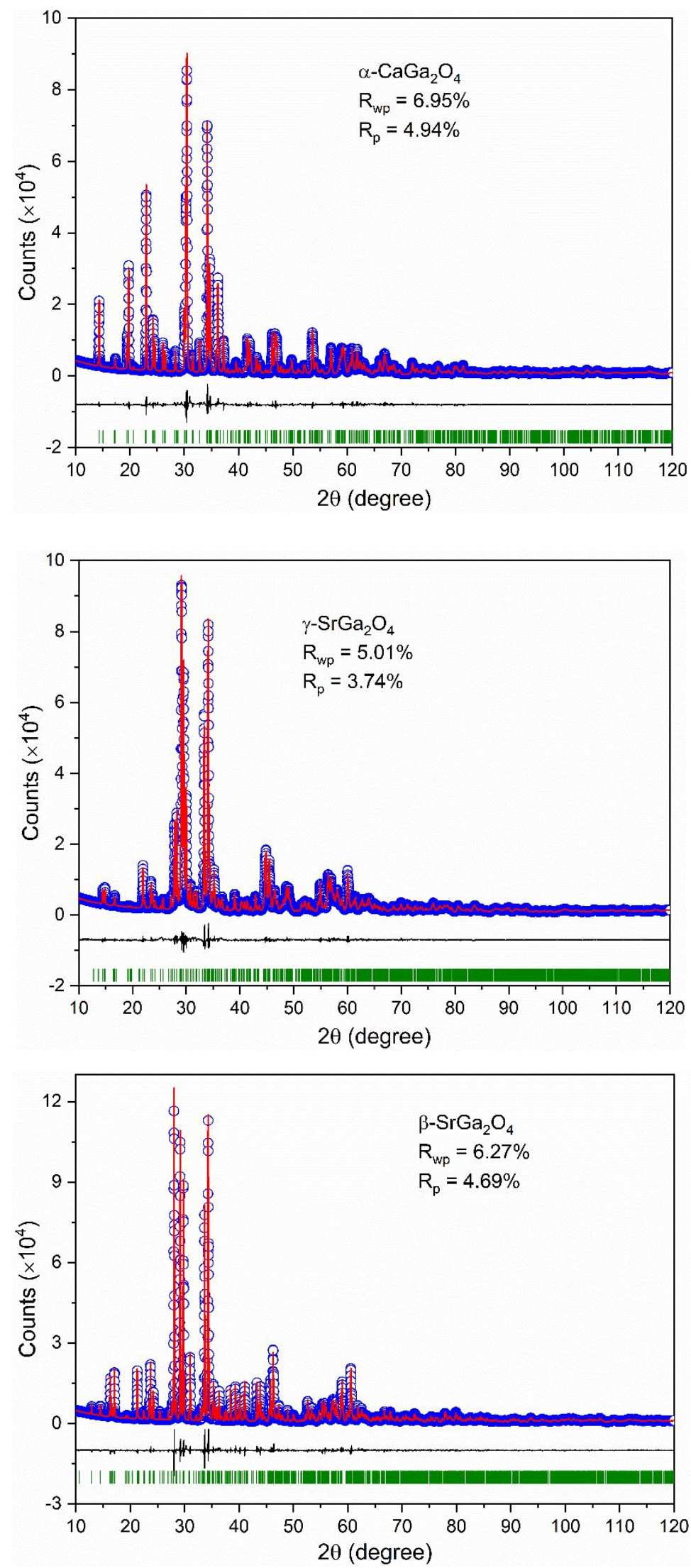

Figure S6. Rietveld refinement patterns for $\alpha-\mathrm{CaGa}_{2} \mathrm{O}_{4}, \beta-\mathrm{SrGa}_{2} \mathrm{O}_{4}$, and $\gamma-\mathrm{SrGa}_{2} \mathrm{O}_{4}$. 

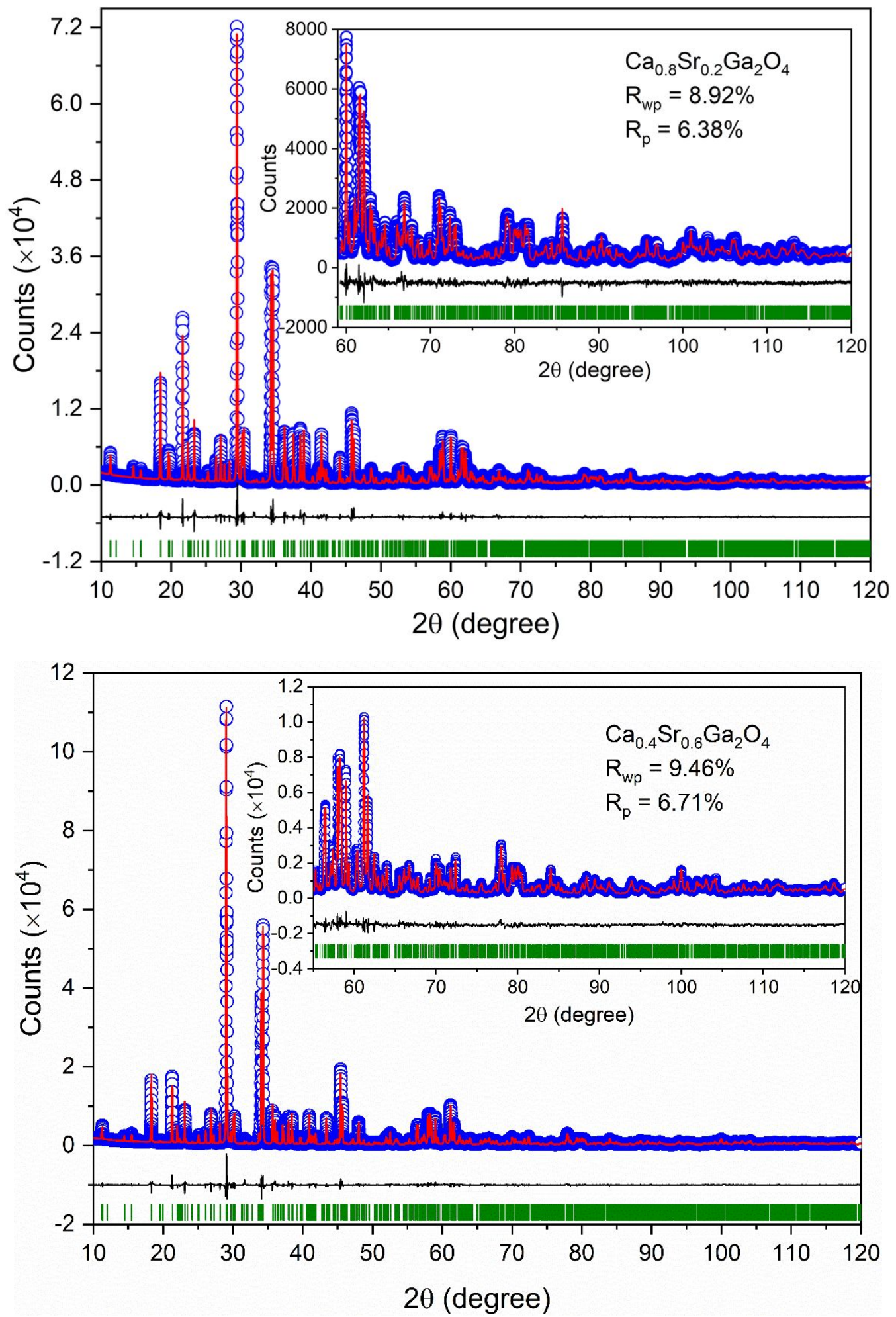

Figure S7. Rietveld refinement plots of $\mathrm{Cu} \mathrm{K} \alpha_{1} \mathrm{XRD}$ data for $\mathrm{Ca}_{1-x} \mathrm{Sr}_{x} \mathrm{Ga}_{2} \mathrm{O}_{4}(x=0.4$ and 0.6). 


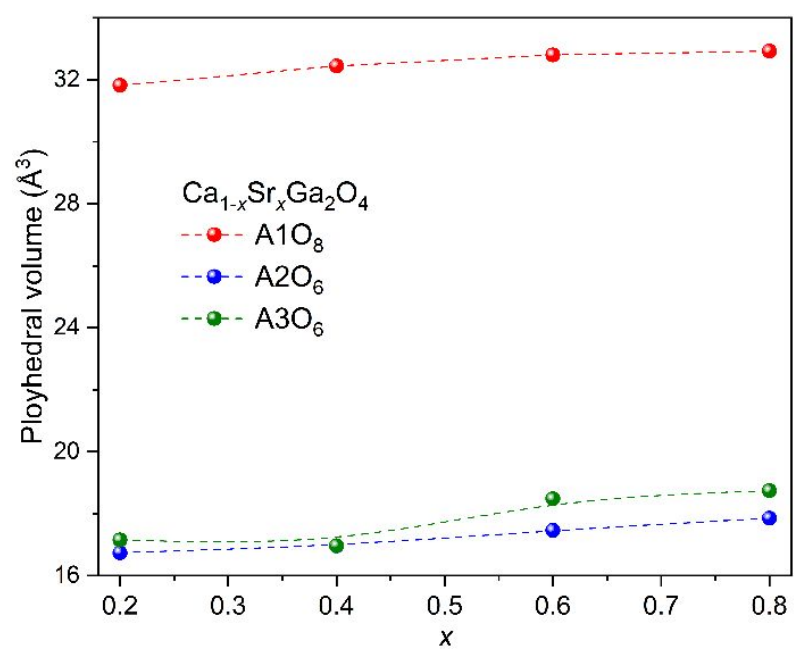

Figure S8. Plots of volumes for $\mathrm{AO}_{\mathrm{n}}$-polyhedra along with Sr-content in the CSGO phases. For $x=$ 0.4 and 0.8 , the parameters are based on neutron powder diffraction data, while the remaining are based on X-ray powder diffraction data.
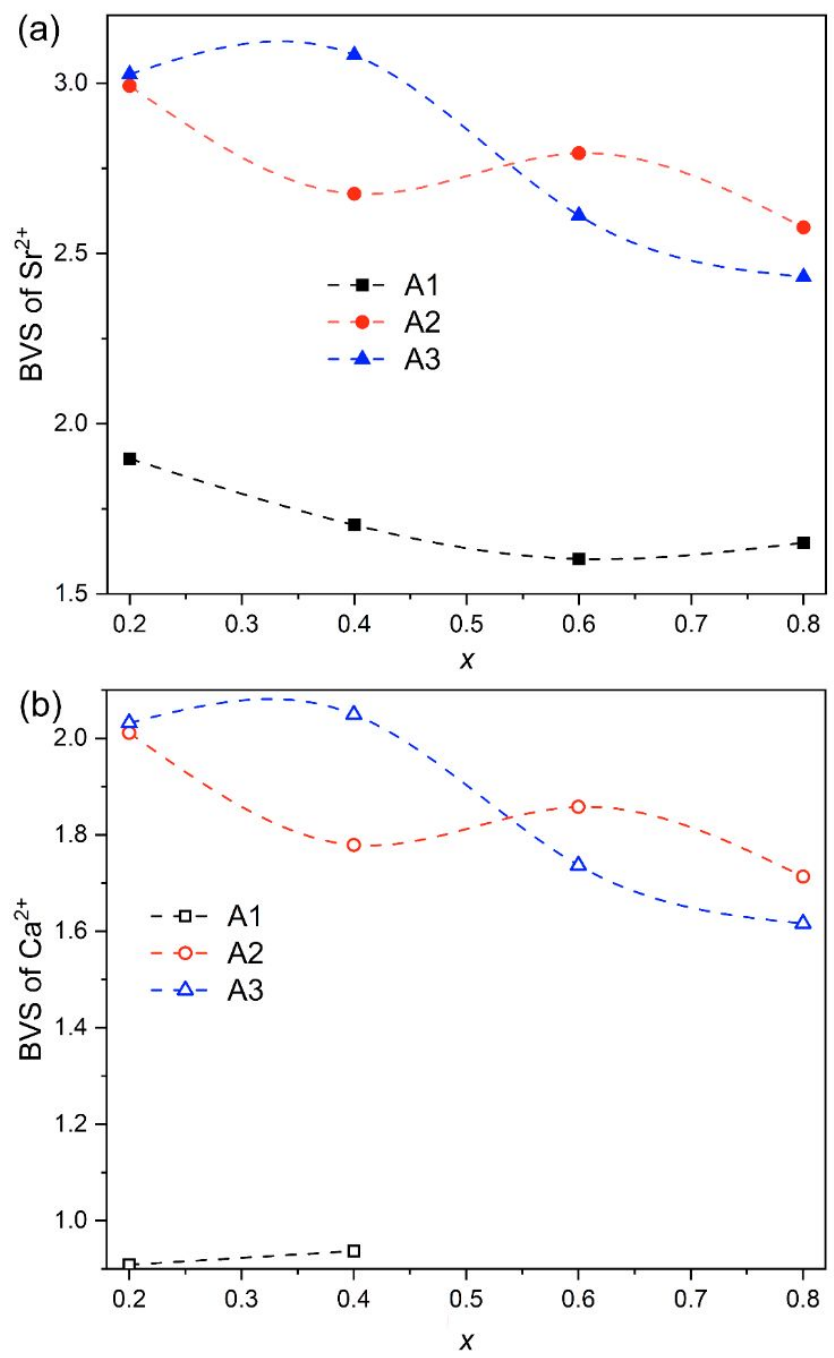

Figure S9. Plots of bond valence sums of $\mathrm{Ca}^{2+} / \mathrm{Sr}^{2+}$ cations at different A-sites against $\mathrm{Sr}^{2+}$-content in 
$\mathrm{Ca}_{1-x} \mathrm{Sr}_{x} \mathrm{Ga}_{2} \mathrm{O}_{4}(x=0.2,0.4,0.6,0.8)$. For $x=0.4$ and 0.8 , the parameters are based on neutron powder diffraction data, while the remaining are based on X-ray powder diffraction data.
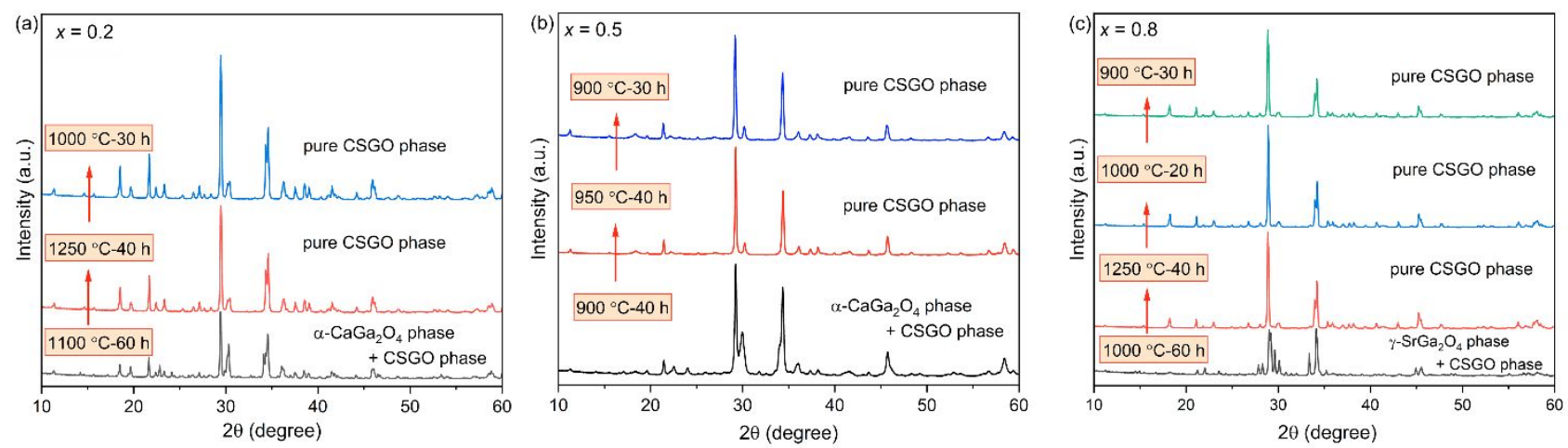

Figure S10. XRD patterns of $\mathrm{Ca}_{1-x} \mathrm{Sr}_{x} \mathrm{Ga}_{2} \mathrm{O}_{4}(x=0.2,0.5$, and 0.8$)$ after a long time annealing at low temperatures.

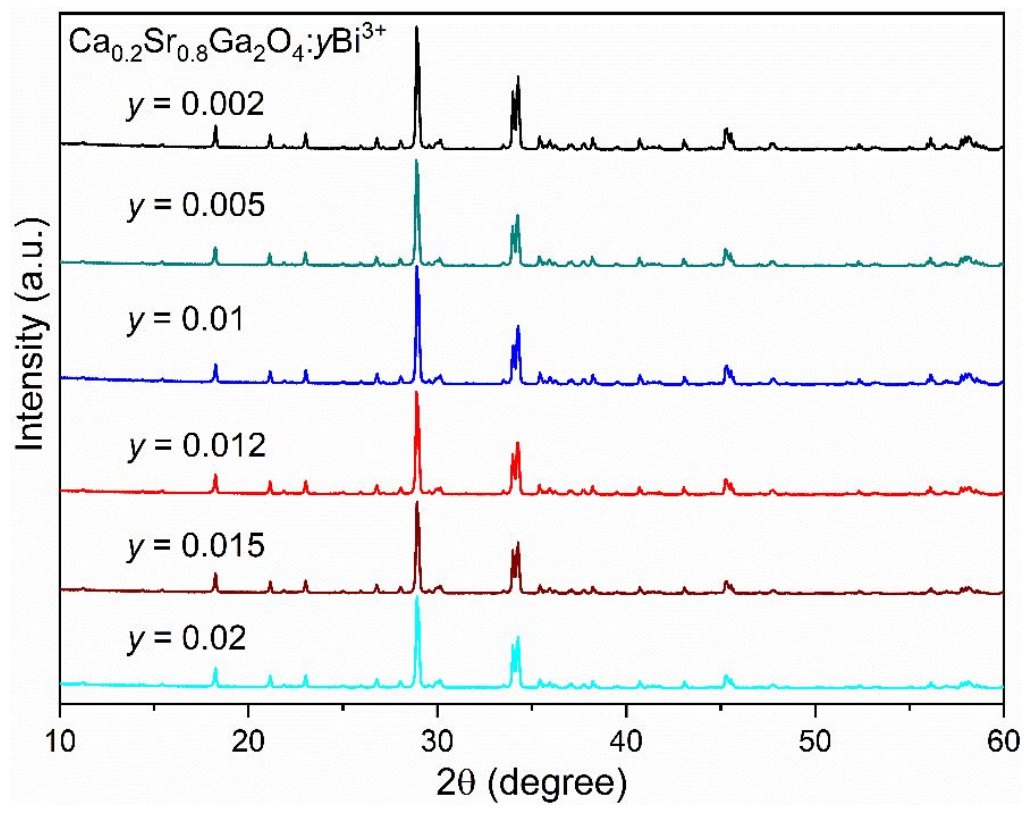




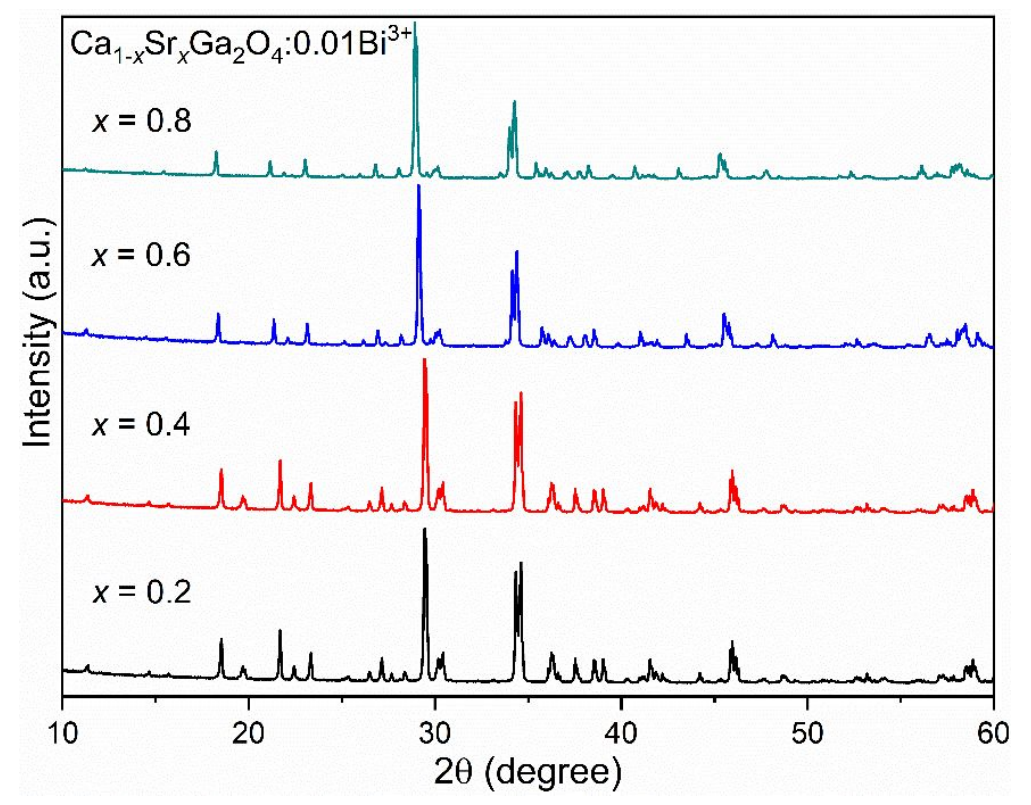

Figure $\mathrm{S} 11$. XRD patterns for $\mathrm{Ca}_{0.2} \mathrm{Sr}_{0.8} \mathrm{Ga}_{2} \mathrm{O}_{4}: y \mathrm{Bi}^{3+}(y=0.002,0.005,0.01,0.012,0.015,0.02)$ and $\mathrm{Ca}_{1-x} \mathrm{Sr}_{x} \mathrm{Ga}_{2} \mathrm{O}_{4}: 0.01 \mathrm{Bi}^{3+}(x=0.2,0.4,0.6,0.8)$. 
(a)
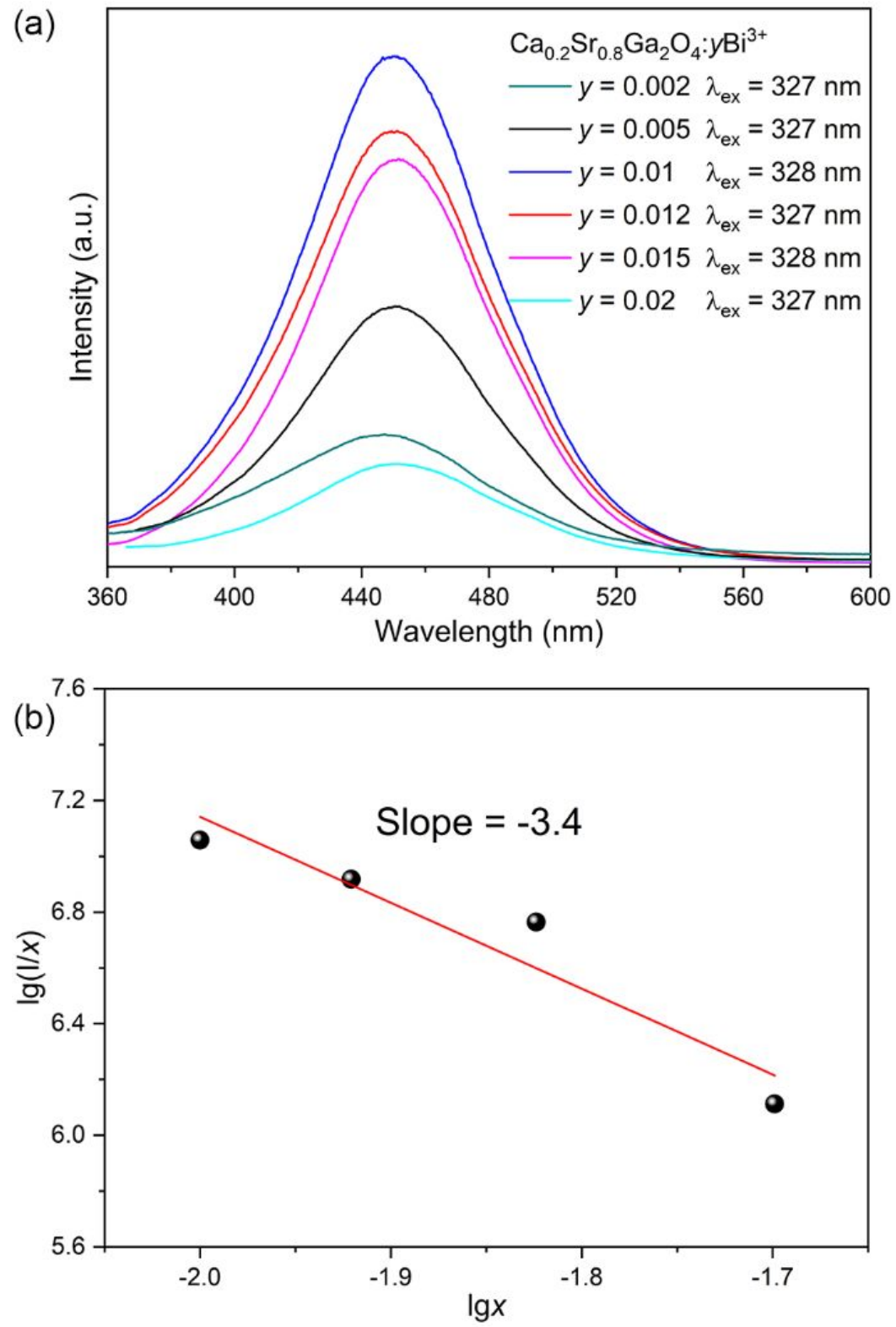

Figure S12. (a) Photoluminescence spectra for $\mathrm{Ca}_{0.2} \mathrm{Sr}_{0.8} \mathrm{Ga}_{2} \mathrm{O}_{4}: \mathrm{yBi}^{3+}(y=0.002,0.005,0.01,0.012$, $0.015,0.02)$. (b) Plots of $\lg (\mathrm{I} / x)$ against $\lg x(x=0.01,0.012,0.015,0.02)$. The red line in (b) represents the fitted line. 


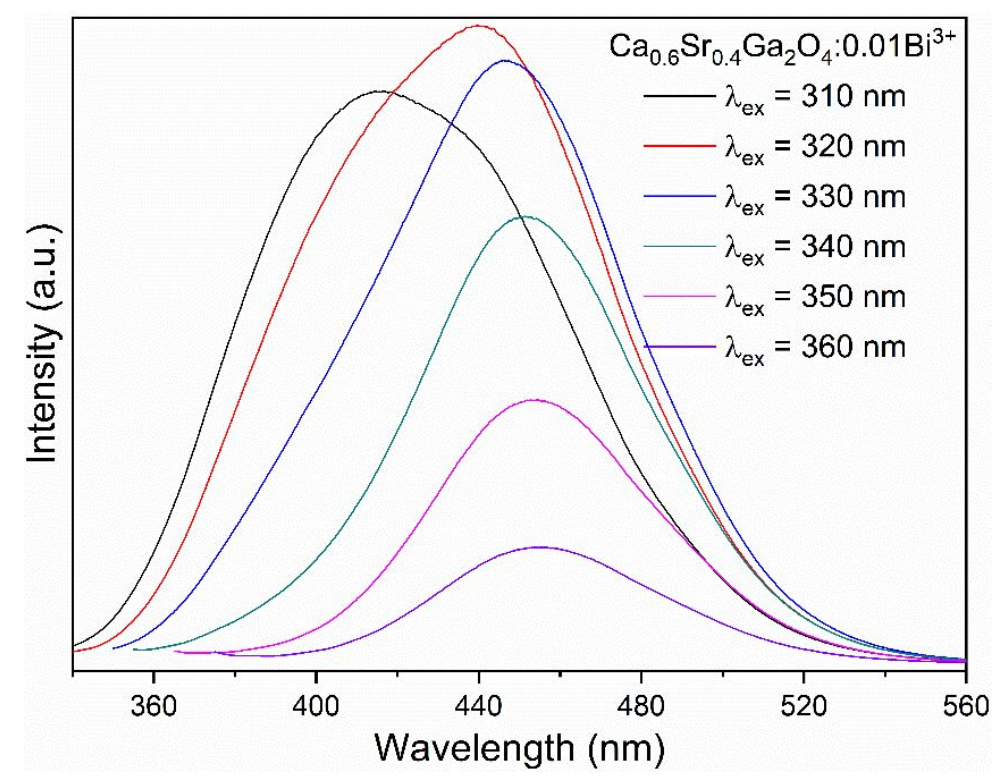

Figure S13. The emission spectra for $\mathrm{Ca}_{0.6} \mathrm{Sr}_{0.4} \mathrm{Ga}_{2} \mathrm{O}_{4}: 0.01 \mathrm{Bi}^{3+}$ excited with different wavelengths.

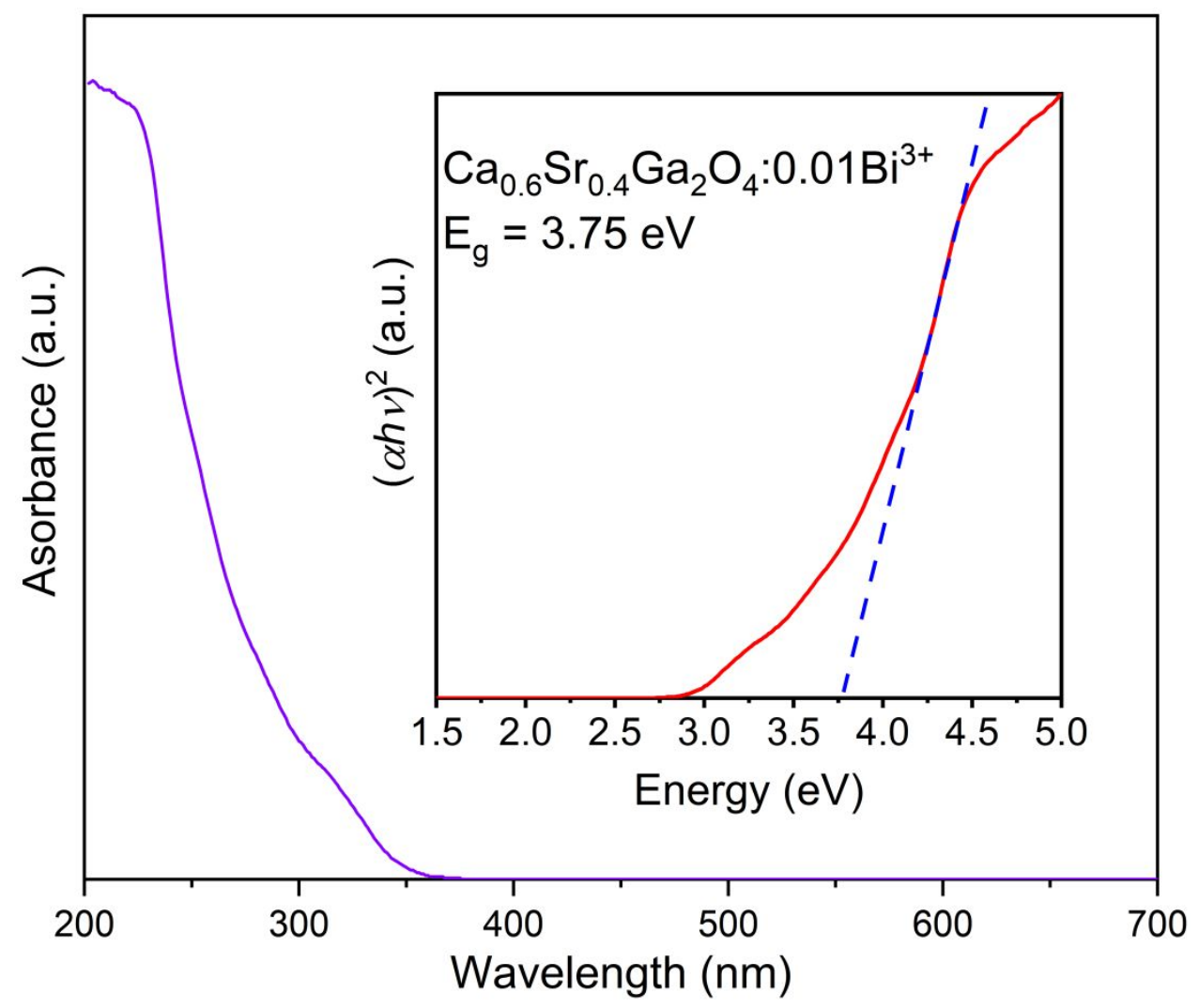

Figure S14. The UV-vis diffuse reflectance spectra (DRS) for $\mathrm{Ca}_{0.6} \mathrm{Sr}_{0.4} \mathrm{Ga}_{2} \mathrm{O}_{4}: 0.01 \mathrm{Bi}^{3+}$. 


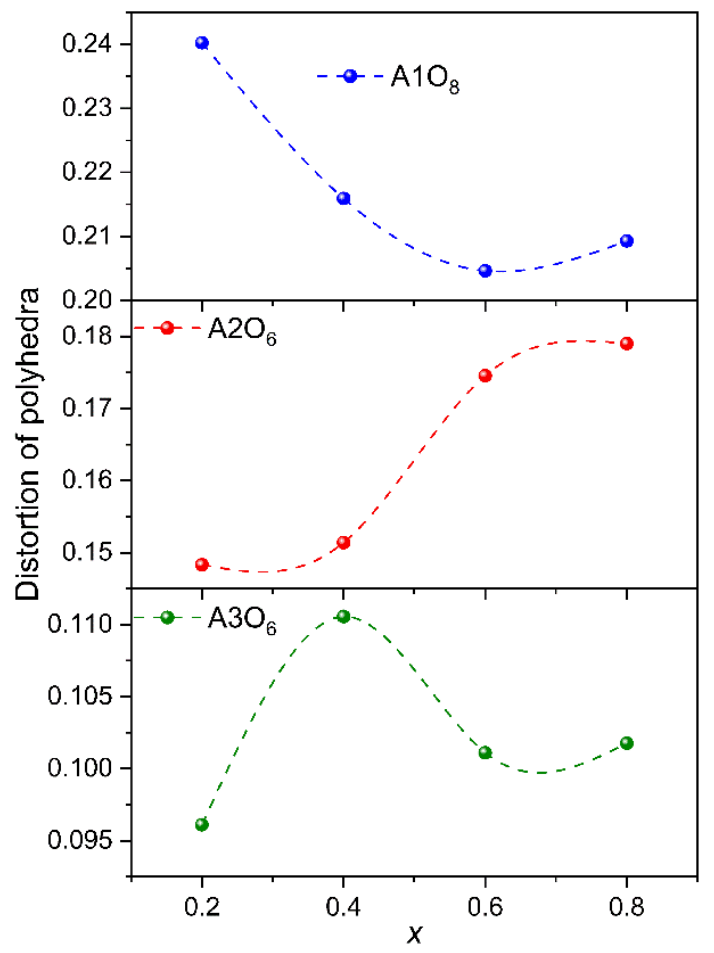

Figure $\mathrm{S} 15$. The calculated distortion indexes for $\mathrm{A}_{10}, \mathrm{~A}_{8} \mathrm{O}_{6}, \mathrm{~A} 3 \mathrm{O}_{6}$ polyhedra in $\mathrm{Ca}_{1-x} \mathrm{Sr}_{x} \mathrm{Ga}_{2} \mathrm{O}_{4}(x$ $=0.2,0.4,0.6$, and 0.8$)$.
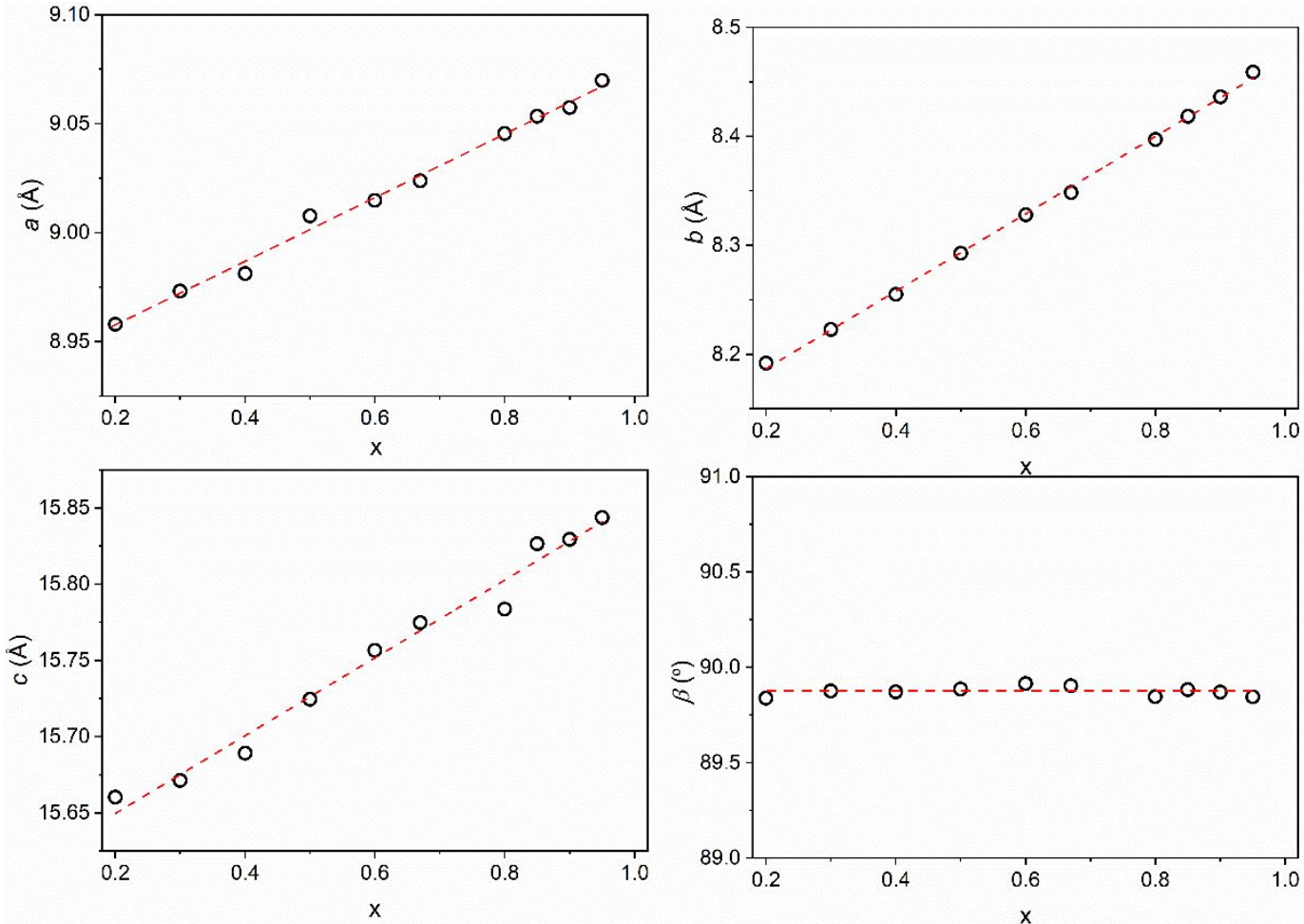

Figure S16. Plots of lattice parameters against $\mathrm{Sr}^{2+}$-content in the CSGO-type $\mathrm{Ca}_{1-x} \mathrm{Sr}_{x} \mathrm{Ga}_{2} \mathrm{O}_{4}$. 
Table S1. The summarization of annealing temperatures for $\mathrm{Ca}_{1-x} \mathrm{Sr}_{x} \mathrm{Ga}_{2} \mathrm{O}_{4}(0<x<1)$.

\begin{tabular}{|c|c|c|c|c|c|c|c|}
\hline \multirow{2}{*}{$\begin{array}{l}\text { Samples } \\
x=0.1\end{array}$} & \multicolumn{7}{|c|}{ Heating process } \\
\hline & $900^{\circ} \mathrm{C} \_40 \mathrm{~h}$ & $950^{\circ} \mathrm{C} \_40 \mathrm{~h}$ & $1000{ }^{\circ} \mathrm{C} \_40 \mathrm{~h}$ & $1050{ }^{\circ} \mathrm{C} \_40 \mathrm{~h}$ & $1080{ }^{\circ} \mathrm{C} \_40 \mathrm{~h}$ & $1250{ }^{\circ} \mathrm{C} \_40 \mathrm{~h}$ & $1300{ }^{\circ} \mathrm{C} \_40 \mathrm{~h}$ \\
\hline & $1335^{\circ} \mathrm{C} \_40 \mathrm{~h}$ & $1350^{\circ} \mathrm{C} \_20 \mathrm{~h}$ & & & & & \\
\hline \multirow[t]{2}{*}{$x=0.125$} & $950^{\circ} \mathrm{C} \_40 \mathrm{~h}$ & $1000^{\circ} \mathrm{C} \_40 \mathrm{~h}$ & $1050^{\circ} \mathrm{C} \_40 \mathrm{~h}$ & $1090{ }^{\circ} \mathrm{C} \_40 \mathrm{~h}$ & $1150^{\circ} \mathrm{C} \_60 \mathrm{~h}$ & $1250^{\circ} \mathrm{C} \_40 \mathrm{~h}$ & $1300{ }^{\circ} \mathrm{C} \_40 \mathrm{~h}$ \\
\hline & $1350^{\circ} \mathrm{C} \_20 \mathrm{~h}$ & & & & & & \\
\hline \multirow[t]{2}{*}{$x=0.15$} & $950^{\circ} \mathrm{C} \_60 \mathrm{~h}$ & $1000^{\circ} \mathrm{C} \_40 \mathrm{~h}$ & $1050^{\circ} \mathrm{C} \_40 \mathrm{~h}$ & $1090^{\circ} \mathrm{C} \_40 \mathrm{~h}$ & $1150^{\circ} \mathrm{C} \_40 \mathrm{~h}$ & $1200^{\circ} \mathrm{C} \_40 \mathrm{~h}$ & $1250^{\circ} \mathrm{C} \_40 \mathrm{~h}$ \\
\hline & $1300^{\circ} \mathrm{C} \_20 \mathrm{~h}$ & $1350^{\circ} \mathrm{C} \_20 \mathrm{~h}$ & & & & & \\
\hline \multirow[t]{2}{*}{$x=0.2$} & $850^{\circ} \mathrm{C} \_40 \mathrm{~h}$ & $900^{\circ} \mathrm{C} \_40 \mathrm{~h}$ & $1000{ }^{\circ} \mathrm{C} \_40 \mathrm{~h}$ & $1050^{\circ} \mathrm{C} \_40 \mathrm{~h}$ & $1150^{\circ} \mathrm{C} \_40 \mathrm{~h}$ & $1200{ }^{\circ} \mathrm{C} \_60 \mathrm{~h}$ & $1250^{\circ} \mathrm{C} \_20 \mathrm{~h}$ \\
\hline & $1300^{\circ} \mathrm{C} \_20 \mathrm{~h}$ & $1350^{\circ} \mathrm{C} \_20 \mathrm{~h}$ & $1400^{\circ} \mathrm{C} \_20 \mathrm{~h}$ & & & & \\
\hline$x=0.3$ & $900^{\circ} \mathrm{C} \_40 \mathrm{~h}$ & $950^{\circ} \mathrm{C} \_40 \mathrm{~h}$ & $1000^{\circ} \mathrm{C} \_60 \mathrm{~h}$ & $1080^{\circ} \mathrm{C} \_20 \mathrm{~h}$ & $1200{ }^{\circ} \mathrm{C} \_20 \mathrm{~h}$ & & \\
\hline \multirow[t]{2}{*}{$x=0.4$} & $750^{\circ} \mathrm{C} \_20 \mathrm{~h}$ & $850^{\circ} \mathrm{C} \_40 \mathrm{~h}$ & $900^{\circ} \mathrm{C} \_40 \mathrm{~h}$ & $950^{\circ} \mathrm{C} \_40 \mathrm{~h}$ & $1000^{\circ} \mathrm{C} \_40 \mathrm{~h}$ & $1050^{\circ} \mathrm{C} \_40 \mathrm{~h}$ & $1080^{\circ} \mathrm{C} \_40 \mathrm{~h}$ \\
\hline & $1200^{\circ} \mathrm{C} \_20 \mathrm{~h}$ & $1250^{\circ} \mathrm{C} \_20 \mathrm{~h}$ & $1300{ }^{\circ} \mathrm{C} \_20 \mathrm{~h}$ & $1350^{\circ} \mathrm{C} \_20 \mathrm{~h}$ & & & \\
\hline$x=0.5$ & $800^{\circ} \mathrm{C} \_40 \mathrm{~h}$ & $850^{\circ} \mathrm{C} \_40 \mathrm{~h}$ & $900^{\circ} \mathrm{C} \_40 \mathrm{~h}$ & $950^{\circ} \mathrm{C} \_40 \mathrm{~h}$ & $1000^{\circ} \mathrm{C} \_20 \mathrm{~h}$ & & \\
\hline \multirow[t]{2}{*}{$x=0.6$} & $850^{\circ} \mathrm{C} \_40 \mathrm{~h}$ & $900^{\circ} \mathrm{C} \_40 \mathrm{~h}$ & $920^{\circ} \mathrm{C} \_40 \mathrm{~h}$ & $950^{\circ} \mathrm{C} \_40 \mathrm{~h}$ & $1000{ }^{\circ} \mathrm{C} \_40 \mathrm{~h}$ & $1050^{\circ} \mathrm{C} \_20 \mathrm{~h}$ & $1080^{\circ} \mathrm{C} \_20 \mathrm{~h}$ \\
\hline & $1200^{\circ} \mathrm{C} \_20 \mathrm{~h}$ & $1250^{\circ} \mathrm{C} \_20 \mathrm{~h}$ & $1300{ }^{\circ} \mathrm{C} \_20 \mathrm{~h}$ & $1350{ }^{\circ} \mathrm{C} \_20 \mathrm{~h}$ & & & \\
\hline$x=0.67$ & $800^{\circ} \mathrm{C} \_40 \mathrm{~h}$ & $850^{\circ} \mathrm{C} \_40 \mathrm{~h}$ & $900^{\circ} \mathrm{C} \_40 \mathrm{~h}$ & $950^{\circ} \mathrm{C} \_40 \mathrm{~h}$ & $1200{ }^{\circ} \mathrm{C} \_20 \mathrm{~h}$ & $1250^{\circ} \mathrm{C} \_20 \mathrm{~h}$ & \\
\hline \multirow[t]{2}{*}{$x=0.8$} & $850^{\circ} \mathrm{C} \_40 \mathrm{~h}$ & $900^{\circ} \mathrm{C} \_40 \mathrm{~h}$ & $950^{\circ} \mathrm{C} \_60 \mathrm{~h}$ & $1000^{\circ} \mathrm{C} \_40 \mathrm{~h}$ & $1050^{\circ} \mathrm{C} \_60 \mathrm{~h}$ & $1080^{\circ} \mathrm{C} \_60 \mathrm{~h}$ & $1200^{\circ} \mathrm{C} \_20 \mathrm{~h}$ \\
\hline & $1250^{\circ} \mathrm{C} \_20 \mathrm{~h}$ & $1300^{\circ} \mathrm{C} \_20 \mathrm{~h}$ & $1350^{\circ} \mathrm{C} \_20 \mathrm{~h}$ & $1400^{\circ} \mathrm{C} \_20 \mathrm{~h}$ & & & \\
\hline$x=0.85$ & $950^{\circ} \mathrm{C} \_40 \mathrm{~h}$ & $1000^{\circ} \mathrm{C} \_40 \mathrm{~h}$ & $1050{ }^{\circ} \mathrm{C} \_60 \mathrm{~h}$ & $1080{ }^{\circ} \mathrm{C} \_40 \mathrm{~h}$ & $1150^{\circ} \mathrm{C} \_40 \mathrm{~h}$ & $1200^{\circ} \mathrm{C} \_40 \mathrm{~h}$ & $1250^{\circ} \mathrm{C} \_40 \mathrm{~h}$ \\
\hline$x=0.875$ & $850^{\circ} \mathrm{C} \_40 \mathrm{~h}$ & $1000{ }^{\circ} \mathrm{C} \_40 \mathrm{~h}$ & $1080{ }^{\circ} \mathrm{C} \_60 \mathrm{~h}$ & $1150{ }^{\circ} \mathrm{C} \_40 \mathrm{~h}$ & $1220^{\circ} \mathrm{C} \_40 \mathrm{~h}$ & $1250^{\circ} \mathrm{C} \_40 \mathrm{~h}$ & $1300^{\circ} \mathrm{C} \_40 \mathrm{~h}$ \\
\hline
\end{tabular}




\begin{tabular}{|c|c|c|c|c|c|c|c|}
\hline \multirow[t]{2}{*}{$x=0.9$} & $900^{\circ} \mathrm{C} \_40 \mathrm{~h}$ & $950^{\circ} \mathrm{C} \_40 \mathrm{~h}$ & $1000^{\circ} \mathrm{C} \_40 \mathrm{~h}$ & $1050^{\circ} \mathrm{C} \_40 \mathrm{~h}$ & $1080^{\circ} \mathrm{C} \_40 \mathrm{~h}$ & $1150^{\circ} \mathrm{C} \_60 \mathrm{~h}$ & $1200^{\circ} \mathrm{C} \_40 \mathrm{~h}$ \\
\hline & $1250^{\circ} \mathrm{C} \_40 \mathrm{~h}$ & $1300^{\circ} \mathrm{C} \_40 \mathrm{~h}$ & $1350^{\circ} \mathrm{C} \_40 \mathrm{~h}$ & $1400^{\circ} \mathrm{C} \_20 \mathrm{~h}$ & & & \\
\hline \multirow[t]{2}{*}{$x=0.95$} & $950^{\circ} \mathrm{C} \_40 \mathrm{~h}$ & $1050^{\circ} \mathrm{C} \_40 \mathrm{~h}$ & $1080^{\circ} \mathrm{C} \_40 \mathrm{~h}$ & $1150^{\circ} \mathrm{C} \_60 \mathrm{~h}$ & $1250^{\circ} \mathrm{C} \_40 \mathrm{~h}$ & $1300^{\circ} \mathrm{C} \_40 \mathrm{~h}$ & $1350^{\circ} \mathrm{C} \_40 \mathrm{~h}$ \\
\hline & $1400^{\circ} \mathrm{C} \_20 \mathrm{~h}$ & & & & & & \\
\hline
\end{tabular}

Table S2. Crystallographic data of $\mathrm{Ca}_{1-x} \mathrm{Sr}_{x} \mathrm{Ga}_{2} \mathrm{O}_{4}(x=0.2,0.4,0.6,0.8)$.

\begin{tabular}{|c|c|c|c|c|}
\hline Source & laboratory X-ray & neutron time of & laboratory X-ray & neutron time \\
\hline & & flight & & flight \\
\hline Chemical formula & $\mathrm{Ca}_{0.8} \mathrm{Sr}_{0.2} \mathrm{Ga}_{2} \mathrm{O}_{4}$ & $\mathrm{Ca}_{0.6} \mathrm{Sr}_{0.4} \mathrm{Ga}_{2} \mathrm{O}_{4}$ & $\mathrm{Ca}_{0.4} \mathrm{Sr}_{0.6} \mathrm{Ga}_{2} \mathrm{O}_{4}$ & $\mathrm{Ca}_{0.2} \mathrm{Sr}_{0.8} \mathrm{Ga}_{2} \mathrm{O}_{4}$ \\
\hline Formula weight & 253.03 & 262.54 & 272.05 & 281.56 \\
\hline \multicolumn{5}{|l|}{$(\mathrm{g} / \mathrm{mol})$} \\
\hline Temperature (K) & 300 & 300 & 300 & 300 \\
\hline \multirow[t]{2}{*}{ Wavelength } & $\mathrm{Cu} \mathrm{K} \alpha 1$ & - & $\mathrm{Cu} \mathrm{K} \alpha 1$ & - \\
\hline & $\lambda=1.54056 \AA$ & & $\lambda=1.54056 \AA$ & \\
\hline Crystal system & Monoclinic & Monoclinic & Monoclinic & Monoclinic \\
\hline Space group & $P 2_{1} / n$ (No. 14$)$ & $P 2_{1} / n$ (No. 14$)$ & $P 2_{1} / n$ (No. 14$)$ & $P 2_{1} / n$ (No. 14$)$ \\
\hline Unit & $a=8.95851(8) \AA$, & $a=8.984(2) \AA$ & $a=9.0151(5) \AA$ & $a=9.047(2) \AA$ \\
\hline \multirow[t]{3}{*}{ dimensions } & $b=8.19242(7) \AA$, & $b=8.259(2) \AA$ & $b=8.32868(7) \AA$, & $b=8.400(2) \AA$ \\
\hline & $c=15.6612(1) \AA$, & $c=15.693(2) \AA$ & $c=15.7590(1) \AA$, & $c=15.806(5) \AA$ \\
\hline & $\beta=89.8444(4)^{\mathrm{o}}$ & $\beta=89.917(4)^{\mathrm{o}}$ & $\beta=89.9213(5)^{\mathrm{o}}$ & $\beta=89.917(2)^{\circ}$ \\
\hline$V\left(\AA^{3}\right)$ & $1149.40(2)$ & $1165.6(5)$ & $1183.24(2)$ & $1200.7(5)$ \\
\hline$Z$ & 12 & 12 & 12 & 12 \\
\hline
\end{tabular}




\begin{tabular}{|c|c|c|c|c|}
\hline$\chi^{2}, R_{\mathrm{p}}, R_{\mathrm{wp}}$ & $2.95,6.25,8.78$ & $3.64,2.95,3.00$ & $3.29,6.71,9.46$ & $3.90,3.07,3.09$ \\
\hline
\end{tabular}

Table S3. The atomic coordinates, occupancies, and isotropic thermal displacement factors for $\mathrm{Ca}_{1-x} \mathrm{Sr}_{x} \mathrm{Ga}_{2} \mathrm{O}_{4}$ obtained from Rietveld refinements against PXRD $(x=0.2$ and 0.6$)$ and PND $(x=0.4$ and 0.8$)$ data.

\begin{tabular}{|c|c|c|c|c|c|}
\hline $\mathrm{Ca}_{0.8} \mathrm{Sr}_{0.2} \mathrm{Ga}_{2} \mathrm{O}_{4}$ & $x$ & $y$ & $z$ & Occ. & $\mathrm{B}_{\text {eq. }}\left(\AA^{2}\right)$ \\
\hline $\mathrm{Sr} 1 / \mathrm{Ca} 1$ & $0.2411(2)$ & $0.0089(3)$ & $0.2513(2)$ & $0.564(4) / 0.436(4)$ & $0.72(4)$ \\
\hline $\mathrm{Ca} 2 / \mathrm{Sr} 2$ & $0.7331(3)$ & $0.4677(5)$ & $0.0727(2)$ & $0.988(7) / 0.012(7)$ & $0.7(1)$ \\
\hline $\mathrm{Ca} 3 / \mathrm{Sr} 3$ & $0.7559(3)$ & $0.0359(5)$ & $0.0871(2)$ & $0.976(8) / 0.024(8)$ & $0.67(9)$ \\
\hline Ga1 & $0.5785(3)$ & $0.3287(3)$ & $0.2378(1)$ & 1 & $0.84(6)$ \\
\hline $\mathrm{Ga} 2$ & $0.0598(3)$ & $0.3353(3)$ & $0.0903(1)$ & 1 & $1.01(7)$ \\
\hline $\mathrm{Ga} 3$ & $0.0991(3)$ & $0.3292(3)$ & $0.4225(2)$ & 1 & $0.661(5)$ \\
\hline $\mathrm{Ga} 4$ & $0.9207(3)$ & $0.2324(3)$ & $0.2674(1)$ & 1 & $0.60(6)$ \\
\hline Ga5 & $0.4365(2)$ & $0.2319(3)$ & $0.4084(1)$ & 1 & $0.12(5)$ \\
\hline $\mathrm{Ga} 6$ & $0.3896(2)$ & $0.2301(4)$ & $0.0807(1)$ & 1 & $0.41(5)$ \\
\hline $\mathrm{O} 1$ & $0.419(1)$ & $0.223(1)$ & $0.1931(6)$ & 1 & $0.43(5)$ \\
\hline $\mathrm{O} 2$ & $0.417(1)$ & $0.736(1)$ & $0.3031(6)$ & 1 & $0.43(5)$ \\
\hline $\mathrm{O} 3$ & $0.244(1)$ & $0.233(1)$ & $0.3611(7)$ & 1 & $0.43(5)$ \\
\hline $\mathrm{O} 4$ & $0.758(1)$ & $0.245(1)$ & $0.1957(6)$ & 1 & $0.43(5)$ \\
\hline $\mathrm{O} 5$ & $0.911(1)$ & $0.235(1)$ & $0.0239(6)$ & 1 & $0.43(5)$ \\
\hline O6 & $0.572(1)$ & $0.261(1)$ & $0.0264(6)$ & 1 & $0.43(5)$ \\
\hline $\mathrm{O} 7$ & $0.555(1)$ & $0.379(1)$ & $0.3564(6)$ & 1 & $0.43(5)$ \\
\hline O8 & $0.256(1)$ & $0.373(1)$ & $0.0474(6)$ & 1 & $0.43(5)$ \\
\hline
\end{tabular}




\begin{tabular}{|c|c|c|c|c|c|}
\hline O9 & $0.935(1)$ & $0.367(1)$ & $0.3590(5)$ & 1 & $0.43(5)$ \\
\hline $\mathrm{O} 10$ & $0.574(1)$ & $0.534(1)$ & $0.1796(5)$ & 1 & $0.43(5)$ \\
\hline O11 & $0.965(1)$ & $0.548(1)$ & $0.1162(5)$ & 1 & $0.43(5)$ \\
\hline $\mathrm{O} 12$ & $0.204(1)$ & $0.532(1)$ & $0.4508(6)$ & 1 & $0.43(5)$ \\
\hline $\mathrm{Ca}_{0.6} \mathrm{Sr}_{0.4} \mathrm{Ga}_{2} \mathrm{O}_{4}$ & $x$ & $y$ & $z$ & Occ. & $\mathrm{B}_{\text {eq. }}\left(\AA^{2}\right)$ \\
\hline $\mathrm{Sr} 1 / \mathrm{Ca} 1$ & $0.2453(3)$ & $0.0040(3)$ & $0.2505(2)$ & $0.886(6) / 0.114(6)$ & $0.73(4)$ \\
\hline $\mathrm{Ca} 2 / \mathrm{Sr} 2$ & $0.7372(4)$ & $0.4693(5)$ & $0.0748(3)$ & $0.83(1) / 0.17(1)$ & $0.7(1)$ \\
\hline $\mathrm{Ca} 3 / \mathrm{Sr} 3$ & $0.7540(4)$ & $0.0362(4)$ & $0.0890(3)$ & $0.86(1) / 0.14(1)$ & $0.84(9)$ \\
\hline Gal & $0.5759(8)$ & $0.3272(6)$ & $0.2390(3)$ & 1 & $0.9(1)$ \\
\hline $\mathrm{Ga} 2$ & $0.0650(8)$ & $0.3326(5)$ & $0.0901(3)$ & 1 & $0.9(1)$ \\
\hline Ga3 & $0.0975(5)$ & $0.3295(6)$ & $0.4217(3)$ & 1 & $1.0(1)$ \\
\hline Ga4 & $0.9203(5)$ & $0.2312(4)$ & $0.2673(2)$ & 1 & $0.64(9)$ \\
\hline Ga5 & $0.4360(4)$ & $0.2337(6)$ & $0.4079(3)$ & 1 & $0.21(1)$ \\
\hline Ga6 & $0.3909(4)$ & $0.2264(6)$ & $0.0802(3)$ & 1 & $0.13(9)$ \\
\hline $\mathrm{O} 1$ & $0.413(2)$ & $0.230(2)$ & $0.194(1)$ & 1 & $0.7(1)$ \\
\hline $\mathrm{O} 2$ & $0.421(2)$ & $0.731(2)$ & $0.305(1)$ & 1 & $0.8(1)$ \\
\hline $\mathrm{O} 3$ & $0.238(2)$ & $0.233(3)$ & $0.367(1)$ & 1 & $1.2(1)$ \\
\hline $\mathrm{O} 4$ & $0.758(2)$ & $0.238(2)$ & $0.1952(9)$ & 1 & $0.3(1)$ \\
\hline O5 & $0.909(1)$ & $0.232(2)$ & $0.0267(9)$ & 1 & $0.7(1)$ \\
\hline O6 & $0.573(2)$ & $0.264(2)$ & $0.025(1)$ & 1 & $0.6(2)$ \\
\hline O7 & $0.566(2)$ & $0.382(2)$ & $0.353(1)$ & 1 & $1.0(1)$ \\
\hline O8 & $0.263(3)$ & $0.373(3)$ & $0.047(1)$ & 1 & $2.3(1)$ \\
\hline
\end{tabular}




\begin{tabular}{|c|c|c|c|c|c|}
\hline O9 & $0.929(2)$ & $0.359(2)$ & $0.3599(9)$ & 1 & $0.3(1)$ \\
\hline $\mathrm{O} 10$ & $0.5678(8)$ & $0.5282(9)$ & $0.1831(4)$ & 1 & $1.2(1)$ \\
\hline O11 & $0.9788(8)$ & $0.537(1)$ & $0.1114(4)$ & 1 & $1.2(1)$ \\
\hline $\mathrm{O} 12$ & $0.1878(8)$ & $0.526(1)$ & $0.4497(4)$ & 1 & $1.6(1)$ \\
\hline $\mathrm{Ca}_{0.4} \mathrm{Sr}_{0.6} \mathrm{Ga}_{2} \mathrm{O}_{4}$ & $x$ & $y$ & $z$ & Occ. & $\mathrm{B}_{\text {eq. }}\left(\AA^{2}\right)$ \\
\hline Sr1 & $0.2403(2)$ & $0.0036(2)$ & $0.2507(2)$ & 1 & $0.93(4)$ \\
\hline $\mathrm{Sr} 2 / \mathrm{Ca} 2$ & $0.7321(3)$ & $0.4739(4)$ & $0.0728(2)$ & $0.37(1) / 0.63(1)$ & $0.91(9)$ \\
\hline $\mathrm{Sr} 3 / \mathrm{Ca} 3$ & $0.7576(3)$ & $0.0317(3)$ & $0.0864(2)$ & $0.43(1) / 0.57(1)$ & $0.88(8)$ \\
\hline Gal & $0.57894(3)$ & $0.3237(3)$ & $0.2388(2)$ & 1 & $0.73(6)$ \\
\hline $\mathrm{Ga} 2$ & $0.0597(3)$ & $0.3324(4)$ & $0.0903(2)$ & 1 & $0.96(7)$ \\
\hline Ga3 & $0.0965(3)$ & $0.3255(4)$ & $0.4222(2)$ & 1 & $1.40(8)$ \\
\hline Ga4 & $0.9209(4)$ & $0.2262(3)$ & $0.2665(2)$ & 1 & $0.80(7)$ \\
\hline Ga5 & $0.4330(3)$ & $0.2288(4)$ & $0.4086(2)$ & 1 & $0.30(6)$ \\
\hline Ga6 & $0.3893(3)$ & $0.2245(4)$ & $0.0802(2)$ & 1 & $0.48(6)$ \\
\hline $\mathrm{O} 1$ & $0.421(1)$ & $0.219(1)$ & $0.1928(8)$ & 1 & $0.68(6)$ \\
\hline $\mathrm{O} 2$ & $0.418(1)$ & $0.729(1)$ & $0.3068(8)$ & 1 & $0.68(6)$ \\
\hline $\mathrm{O} 3$ & $0.245(1)$ & $0.243(2)$ & $0.3656(7)$ & 1 & $0.68(6)$ \\
\hline $\mathrm{O} 4$ & $0.759(2)$ & $0.244(1)$ & $0.1962(7)$ & 1 & $0.68(6)$ \\
\hline O5 & $0.912(1)$ & $0.234(1)$ & $0.0231(8)$ & 1 & $0.68(6)$ \\
\hline O6 & $0.571(1)$ & $0.261(1)$ & $0.0273(7)$ & 1 & $0.68(6)$ \\
\hline O7 & $0.551(1)$ & $0.375(1)$ & $0.3558(7)$ & 1 & $0.68(6)$ \\
\hline O8 & $0.253(1)$ & $0.363(1)$ & $0.0433(8)$ & 1 & $0.68(6)$ \\
\hline
\end{tabular}




\begin{tabular}{|c|c|c|c|c|c|}
\hline O9 & $0.932(1)$ & $0.356(1)$ & $0.3590(7)$ & 1 & $0.68(6)$ \\
\hline $\mathrm{O} 10$ & $0.577(1)$ & $0.524(1)$ & $0.1803(6)$ & 1 & $0.68(6)$ \\
\hline O11 & $0.968(1)$ & $0.543(1)$ & $0.1213(6)$ & 1 & $0.68(6)$ \\
\hline $\mathrm{O} 12$ & $0.202(1)$ & $0.529(2)$ & $0.4460(7)$ & 1 & $0.68(6)$ \\
\hline $\mathrm{Ca}_{0.2} \mathrm{Sr}_{0.8} \mathrm{Ga}_{2} \mathrm{O}_{4}$ & $x$ & $y$ & $z$ & Occ. & $\mathrm{B}_{\text {eq. }}\left(\AA^{2}\right)$ \\
\hline Sr1 & $0.2401(2)$ & $0.0033(3)$ & $0.2493(2)$ & 1 & $0.73(4)$ \\
\hline $\mathrm{Sr} 2 / \mathrm{Ca} 2$ & $0.7316(3)$ & $0.4755(3)$ & $0.0731(2)$ & $0.70(1) / 0.30(1)$ & $1.25(8)$ \\
\hline $\mathrm{Sr} 3 / \mathrm{Ca} 3$ & $0.7586(3)$ & $0.0297(3)$ & $0.0873(2)$ & $0.70(1) / 0.30(1)$ & $0.91(7)$ \\
\hline Ga1 & $0.5789(7)$ & $0.3269(5)$ & $0.2373(3)$ & 1 & $0.89(9)$ \\
\hline $\mathrm{Ga} 2$ & $0.0624(7)$ & $0.3305(7)$ & $0.0910(4)$ & 1 & $0.62(9)$ \\
\hline $\mathrm{Ga} 3$ & $0.0921(6)$ & $0.3252(7)$ & $0.4218(4)$ & 1 & $1.2(1)$ \\
\hline $\mathrm{Ga} 4$ & $0.9218(5)$ & $0.2237(4)$ & $0.2664(3)$ & 1 & $1.0(1)$ \\
\hline Ga5 & $0.4301(4)$ & $0.2241(5)$ & $0.4088(3)$ & 1 & $0.5(1)$ \\
\hline Ga6 & $0.3885(5)$ & $0.2160(5)$ & $0.0820(3)$ & 1 & $0.2(1)$ \\
\hline $\mathrm{O} 1$ & $0.418(2)$ & $0.213(1)$ & $0.198(1)$ & 1 & $1.5(2)$ \\
\hline $\mathrm{O} 2$ & $0.415(2)$ & $0.740(2)$ & $0.304(9)$ & 1 & $0.4(1)$ \\
\hline $\mathrm{O} 3$ & $0.243(2)$ & $0.227(2)$ & $0.369(1)$ & 1 & $1.3(1)$ \\
\hline $\mathrm{O} 4$ & $0.755(2)$ & $0.226(2)$ & $0.198(1)$ & 1 & $0.7(1)$ \\
\hline O5 & $0.913(2)$ & $0.239(2)$ & $0.0268(9)$ & 1 & $0.4(1)$ \\
\hline O6 & $0.566(2)$ & $0.263(2)$ & $0.025(1)$ & 1 & $0.3(1)$ \\
\hline O7 & $0.557(2)$ & $0.368(2)$ & $0.356(1)$ & 1 & $1.5(2)$ \\
\hline O8 & $0.243(1)$ & $0.363(2)$ & $0.039(1)$ & 1 & $1.5(2)$ \\
\hline
\end{tabular}




\begin{tabular}{llllll}
\hline O9 & $0.923(2)$ & $0.342(2)$ & $0.3616(6)$ & 1 & $0.8(1)$ \\
O10 & $0.556(1)$ & $0.520(1)$ & $0.1885(5)$ & 1 & $1.6(1)$ \\
O11 & $0.9866(9)$ & $0.527(1)$ & $0.1131(5)$ & 1 & $1.6(2)$ \\
O12 & $0.1840(9)$ & $0.520(1)$ & $0.4410(4)$ & 1 & $1.1(1)$ \\
\hline
\end{tabular}

Table S4. Selected interatomic distances $(\AA)$ in $\mathrm{Ca}_{1-x} \mathrm{Sr}_{x} \mathrm{Ga}_{2} \mathrm{O}_{4}$ obtained from Rietveld refinement against PXRD $(x=0.2$ and 0.6$)$ and PND $(x=0.4$ and 0.8$)$ data.

\begin{tabular}{|c|c|c|c|c|c|}
\hline \multicolumn{6}{|c|}{$\mathrm{Ca}_{0.8} \mathrm{Sr}_{0.2} \mathrm{Ga}_{2} \mathrm{O}_{4}$} \\
\hline $\mathrm{Sr} 1 / \mathrm{Ca} 1-\mathrm{O} 2$ & $2.49(1)$ & $\mathrm{Ca} 3-\mathrm{O} 7$ & $2.31(1)$ & Ga3-O6 & $1.81(1)$ \\
\hline $\mathrm{Sr} 1 / \mathrm{Ca} 1-\mathrm{O} 3$ & $2.51(1)$ & $\mathrm{Ca} 3-\mathrm{O} 9$ & $2.36(1)$ & Ga3-O12 & $1.96(1)$ \\
\hline $\mathrm{Sr} 1 / \mathrm{Ca} 1-\mathrm{O} 1$ & $2.54(1)$ & $\mathrm{Ca} 3-\mathrm{O} 5$ & $2.36(1)$ & Ga4-O9 & $1.814(9)$ \\
\hline $\mathrm{Sr} 1 / \mathrm{Ca} 1-\mathrm{O} 2$ & $2.86(1)$ & $\mathrm{Ca} 3-\mathrm{O} 4$ & $2.42(1)$ & $\mathrm{Ga} 4-\mathrm{O} 2$ & $1.82(1)$ \\
\hline $\mathrm{Sr} 1 / \mathrm{Ca} 1-\mathrm{O} 3$ & $2.87(1)$ & Ca3-O6 & $2.66(1)$ & Ga4-O10 & $1.83(1)$ \\
\hline $\mathrm{Sr} 1 / \mathrm{Ca} 1-\mathrm{O} 1$ & $2.88(1)$ & Ga1-O1 & $1.82(1)$ & Ga4-O4 & $1.85(1)$ \\
\hline $\mathrm{Sr} 1 / \mathrm{Ca} 1-\mathrm{O} 10$ & $3.022(9)$ & Ga1-O4 & $1.87(1)$ & Ga5-O11 & $1.79(1)$ \\
\hline $\mathrm{Sr} 1 / \mathrm{Ca} 1-\mathrm{O} 12$ & $3.206(9)$ & Ga1-O10 & $1.91(1)$ & Ga5-O7 & $1.799(9)$ \\
\hline $\mathrm{Ca} 2-\mathrm{O} 10$ & $2.266(9)$ & Ga1-O7 & $1.92(1)$ & Ga5-O5 & $1.84(1)$ \\
\hline $\mathrm{Ca} 2-\mathrm{O} 11$ & $2.289(9)$ & $\mathrm{Ga} 2-\mathrm{O} 2$ & $1.87(1)$ & Ga5-O3 & $1.88(1)$ \\
\hline $\mathrm{Ca} 2-\mathrm{O} 8$ & $2.29(1)$ & Ga2-O5 & $1.88(1)$ & Ga6-O8 & $1.75(1)$ \\
\hline $\mathrm{Ca} 2-\mathrm{O} 6$ & $2.34(1)$ & Ga2-O8 & $1.91(1)$ & Ga6-O1 & $1.78(1)$ \\
\hline $\mathrm{Ca} 2-\mathrm{O} 5$ & $2.596(9)$ & $\mathrm{Ga} 2-\mathrm{O} 11$ & $1.98(1)$ & Ga6-O6 & $1.86(9)$ \\
\hline $\mathrm{Ca} 2-\mathrm{O} 4$ & $2.67(1)$ & Ga3-O3 & $1.795(9)$ & Ga6-O12 & $1.895(9)$ \\
\hline $\mathrm{Ca} 3-\mathrm{O} 12$ & $2.26(1)$ & Ga3-O9 & $1.81(1)$ & & \\
\hline \multicolumn{6}{|c|}{$\mathrm{Ca}_{0.6} \mathrm{Sr}_{0.4} \mathrm{Ga}_{2} \mathrm{O}_{4}$} \\
\hline $\mathrm{Sr} 1 / \mathrm{Ca} 1-\mathrm{O} 2$ & $2.55(2)$ & $\mathrm{Sr} 3 / \mathrm{Ca} 3-\mathrm{O} 7$ & $2.25(2)$ & Ga3-O9 & $1.83(2)$ \\
\hline $\mathrm{Sr} 1 / \mathrm{Ca} 1-\mathrm{O} 3$ & $2.63(2)$ & $\mathrm{Sr} 3 / \mathrm{Ca} 3-\mathrm{O} 5$ & $2.35(2)$ & Ga3-O12 & $1.96(2)$ \\
\hline $\mathrm{Sr} 1 / \mathrm{Ca} 1-\mathrm{O} 1$ & $2.55(2)$ & $\mathrm{Sr} 3 / \mathrm{Ca} 3-\mathrm{O} 9$ & $2.35(2)$ & Ga4-O9 & $1.80(2)$ \\
\hline $\mathrm{Sr} 1 / \mathrm{Ca} 1-\mathrm{O} 1$ & $2.81(2)$ & $\mathrm{Sr} 3 / \mathrm{Ca} 3-\mathrm{O} 4$ & $2.36(2)$ & Ga4-O10 & $1.85(2)$ \\
\hline
\end{tabular}




\begin{tabular}{|c|c|c|c|c|c|}
\hline $\mathrm{Sr} 1 / \mathrm{Ca} 1-\mathrm{O} 3$ & $2.90(2)$ & $\mathrm{Sr} 3 / \mathrm{Ca} 3-\mathrm{O} 6$ & $2.69(2)$ & Ga4-O4 & $1.85(2)$ \\
\hline Sr1/Ca1-O2 & $2.88(2)$ & Ga1-O1 & $1.83(2)$ & Ga4-O2 & $1.82(2)$ \\
\hline Sr1/Ca1-O10 & $3.09(2)$ & Ga1-O7 & $1.86(2)$ & Ga5-O11 & $1.83(2)$ \\
\hline $\mathrm{Sr} 1 / \mathrm{Ca} 1-\mathrm{O} 12$ & $3.13(2)$ & Ga1-O4 & $1.90(2)$ & Ga5-O5 & $1.90(2)$ \\
\hline $\mathrm{Sr} 2 / \mathrm{Ca} 2-\mathrm{O} 10$ & $2.25(2)$ & Ga1-O10 & $1.99(2)$ & Ga5-O7 & $1.90(2)$ \\
\hline $\mathrm{Sr} 2 / \mathrm{Ca} 2-\mathrm{O} 8$ & $2.31(2)$ & $\mathrm{Ga} 2-\mathrm{O} 2$ & $1.84(2)$ & Ga5-O3 & $1.90(2)$ \\
\hline $\mathrm{Sr} 2 / \mathrm{Ca} 2-\mathrm{O} 11$ & $2.27(2)$ & Ga2-O5 & $1.90(2)$ & Ga6-O8 & $1.75(2)$ \\
\hline $\mathrm{Sr} 2 / \mathrm{Ca} 2-\mathrm{O} 6$ & $2.38(2)$ & Ga2-O8 & $1.96(2)$ & Ga6-O1 & $1.81(2)$ \\
\hline $\mathrm{Sr} 2 / \mathrm{Ca} 2-\mathrm{O} 5$ & $2.61(2)$ & Ga2-O11 & $1.93(2)$ & Ga6-O6 & $1.87(2)$ \\
\hline $\mathrm{Sr} 2 / \mathrm{Ca} 2-\mathrm{O} 4$ & $2.70(2)$ & Ga3-O3 & $1.74(2)$ & Ga6-O12 & $1.91(2)$ \\
\hline $\mathrm{Sr} 3 / \mathrm{Ca} 3-\mathrm{O} 12$ & $2.34(2)$ & Ga3-O6 & $1.78(2)$ & & \\
\hline & & $\mathrm{Ca}_{0.4} \mathrm{Sr}_{0.6} \mathrm{G}$ & $\mathbf{O}_{4}$ & & \\
\hline Sr1-O2 & $2.51(1)$ & $\mathrm{Sr} 3 / \mathrm{Ca} 3-\mathrm{O} 7$ & $2.38(1)$ & Ga3-O9 & $1.82(1)$ \\
\hline Sr1-O1 & $2.56(1)$ & $\mathrm{Sr} 3 / \mathrm{Ca} 3-\mathrm{O} 9$ & $2.41(1)$ & Ga3-O12 & $1.97(1)$ \\
\hline Sr1-O3 & $2.67(1)$ & $\mathrm{Sr} 3 / \mathrm{Ca} 3-\mathrm{O} 5$ & $2.41(1)$ & Ga4-O9 & $1.81(1)$ \\
\hline Sr1-O3 & $2.84(1)$ & $\mathrm{Sr} 3 / \mathrm{Ca} 3-\mathrm{O} 4$ & $2.49(1)$ & Ga4-O4 & $1.84(1)$ \\
\hline Sr1-O1 & 2.92(1) & $\mathrm{Sr} 3 / \mathrm{Ca} 3-\mathrm{O} 6$ & $2.73(1)$ & Ga4-O2 & $1.87(1)$ \\
\hline Sr1-O2 & $2.92(1)$ & Ga1-O1 & $1.84(1)$ & $\mathrm{Ga} 4-\mathrm{O} 10$ & $1.88(1)$ \\
\hline Sr1-O10 & $3.04(1)$ & Ga1-O4 & $1.86(1)$ & Ga5-O7 & $1.81(1)$ \\
\hline $\mathrm{Sr} 1-\mathrm{O} 12$ & $3.14(1)$ & Ga1-O7 & $1.89(1)$ & Ga5-O11 & $1.84(1)$ \\
\hline $\mathrm{Sr} 2 / \mathrm{Ca} 2-\mathrm{O} 10$ & $2.25(1)$ & Ga1-O10 & $1.90(1)$ & Ga5-O3 & $1.84(1)$ \\
\hline $\mathrm{Sr} 2 / \mathrm{Ca} 2-\mathrm{O} 8$ & $2.30(1)$ & $\mathrm{Ga} 2-\mathrm{O} 2$ & $1.85(1)$ & Ga5-O5 & $1.85(1)$ \\
\hline $\mathrm{Sr} 2 / \mathrm{Ca} 2-\mathrm{O} 11$ & $2.33(1)$ & Ga2-O5 & $1.88(1)$ & Ga6-O8 & $1.77(1)$ \\
\hline $\mathrm{Sr} 2 / \mathrm{Ca} 2-\mathrm{O} 6$ & $2.42(1)$ & Ga2-O8 & $1.92(1)$ & Ga6-O1 & $1.80(1)$ \\
\hline $\mathrm{Sr} 2 / \mathrm{Ca} 2-\mathrm{O} 5$ & $2.69(1)$ & Ga2-O11 & $1.99(1)$ & Ga6-O6 & $1.85(1)$ \\
\hline $\mathrm{Sr} 2 / \mathrm{Ca} 2-\mathrm{O} 4$ & $2.75(1)$ & Ga3-O3 & $1.75(1)$ & Ga6-O12 & $1.87(1)$ \\
\hline $\mathrm{Sr} 3 / \mathrm{Ca} 3-\mathrm{O} 12$ & $2.33(1)$ & Ga3-O6 & $1.80(1)$ & & \\
\hline \multicolumn{6}{|c|}{$\mathrm{Ca}_{0.2} \mathrm{Sr}_{0.8} \mathrm{Ga}_{2} \mathrm{O}_{4}$} \\
\hline Sr1-O1 & $2.52(1)$ & $\mathrm{Sr} 3 / \mathrm{Ca} 3-\mathrm{O} 12$ & $2.44(1)$ & Ga3-O9 & $1.82(2)$ \\
\hline
\end{tabular}




\begin{tabular}{|c|c|c|c|c|c|}
\hline $\mathrm{Sr} 1-\mathrm{O} 2$ & $2.60(1)$ & $\mathrm{Sr} 3 / \mathrm{Ca} 3-\mathrm{O} 9$ & $2.41(2)$ & Ga3-O12 & $1.93(2)$ \\
\hline Sr1-O3 & $2.66(2)$ & $\mathrm{Sr} 3 / \mathrm{Ca} 3-\mathrm{O} 5$ & $2.44(1)$ & Ga4-O10 & $1.88(2)$ \\
\hline $\mathrm{Sr} 1-\mathrm{O} 2$ & $2.87(2)$ & $\mathrm{Sr} 3 / \mathrm{Ca} 3-\mathrm{O} 4$ & $2.41(2)$ & Ga4-O9 & $1.83(2)$ \\
\hline Sr1-O1 & $2.95(2)$ & $\mathrm{Sr} 3 / \mathrm{Ca} 3-\mathrm{O} 6$ & $2.80(2)$ & Ga4-O4 & $1.86(2)$ \\
\hline $\mathrm{Sr} 1-\mathrm{O} 10$ & $2.97(2)$ & Ga1-O1 & $1.85(2)$ & $\mathrm{Ga} 4-\mathrm{O} 2$ & $1.83(2)$ \\
\hline $\mathrm{Sr} 1-\mathrm{O} 3$ & $2.99(2)$ & Ga1-O4 & $1.90(2)$ & $\mathrm{Ga} 5-\mathrm{O} 7$ & $1.86(2)$ \\
\hline $\mathrm{Sr} 1-\mathrm{O} 12$ & $3.01(2)$ & Ga1-O7 & $1.88(1)$ & Ga5-O11 & $1.86(2)$ \\
\hline $\mathrm{Sr} 2 / \mathrm{Ca} 2-\mathrm{O} 8$ & $2.24(2)$ & Ga1-O10 & $1.91(2)$ & $\mathrm{Ga} 5-\mathrm{O} 3$ & $1.82(2)$ \\
\hline $\mathrm{Sr} 2 / \mathrm{Ca} 2-\mathrm{O} 11$ & $2.35(1)$ & $\mathrm{Ga} 2-\mathrm{O} 5$ & $1.86(1)$ & Ga5-O5 & $1.90(2)$ \\
\hline $\mathrm{Sr} 2 / \mathrm{Ca} 2-\mathrm{O} 10$ & $2.33(1)$ & $\mathrm{Ga} 2-\mathrm{O} 2$ & $1.83(2)$ & Ga6-O8 & $1.88(2)$ \\
\hline $\mathrm{Sr} 2 / \mathrm{Ca} 2-\mathrm{O} 6$ & $2.45(1)$ & $\mathrm{Ga} 2-\mathrm{O} 8$ & $1.92(2)$ & Ga6-O1 & $1.85(2)$ \\
\hline $\mathrm{Sr} 2 / \mathrm{Ca} 2-\mathrm{O} 5$ & $2.68(1)$ & Ga2-O11 & $1.94(2)$ & Ga6-O12 & $1.84(2)$ \\
\hline $\mathrm{Sr} 2 / \mathrm{Ca} 2-\mathrm{O} 4$ & $2.89(2)$ & Ga3-O3 & $1.77(2)$ & Ga6-O6 & $1.89(2)$ \\
\hline $\mathrm{Sr} 3 / \mathrm{Ca} 3-\mathrm{O} 7$ & $2.33(2)$ & Ga3-O6 & $1.80(2)$ & & \\
\hline
\end{tabular}

Table S5. Lattice parameters for $\gamma-\mathrm{SrGa}_{2} \mathrm{O}_{4}$-type $\mathrm{Ca}_{1-x} \mathrm{Sr}_{x} \mathrm{Ga}_{2} \mathrm{O}_{4}(x=0.8,0.85,0.90,0.95)$ phases obtained from Le-bailing fitting.

\begin{tabular}{lllll}
\hline$x$ & $a(\AA)$ & $b(\AA)$ & $c(\AA)$ & $\beta\left({ }^{\circ}\right)$ \\
\hline$x=0.8$ & $8.047(4)$ & $10.712(5)$ & $9.015(1)$ & $91.58(1)$ \\
$x=0.85$ & $8.063(1)$ & $10.726(1)$ & $9.027(1)$ & $91.628(7)$ \\
$x=0.9$ & $8.080(2)$ & $10.731(2)$ & $9.027(2)$ & $91.788(5)$ \\
$x=0.95$ & $8.0941(9)$ & $10.747(1)$ & $9.0443(8)$ & $91.567(4)$ \\
$x=1.0$ & $8.1117(3)$ & $10.7566(4)$ & $9.0529(3)$ & $91.540(7)$ \\
\hline
\end{tabular}

